\title{
Meta-analysis of excision repair cross complementary gene 1 (ERCC1) expression and platinum chemosensitivity in patients with ovarian cancer
}

\author{
Jiaohong $\mathrm{Wu}^{1}$, Renliang $\mathrm{Li}^{1}$, Xinyan Chen ${ }^{1}$, Cong Chen ${ }^{1}$, Haitang Chen ${ }^{1}$, Bidong $\mathrm{Ma}^{2}$ \\ ${ }^{1}$ Department of Obstetrics and Gynecology, Wenzhou People's Hospital, Wenzhou, China; ${ }^{2}$ Department of Oncology, Wenzhou Traditional Chinese \\ Medicine Hospital, Wenzhou, China \\ Contributions: (I) Conception and design: J Wu, B Ma; (II) Administrative support: B Ma; (III) Provision of study materials or patients: X Chen, C \\ Chen, H Chen; (IV) Collection and assembly of data: J Wu, R Li, X Chen, C Chen, H Chen; (V) Data analysis and interpretation: J Wu, R Li, B Ma; \\ (VI) Manuscript writing: All authors; (VII) Final approval of manuscript: All authors. \\ Correspondence to: Bidong Ma. Department of Oncology, Wenzhou Traditional Chinese Medicine Hospital, Wenzhou 325000, China. \\ Email: mabidong1234@126.com.
}

Background: Ovarian cancer is one of the most common malignant tumors in women. Due to the lack of
typical clinical symptoms and ineffective screening methods for early ovarian cancer, 60-70\% of patients with
ovarian cancer are diagnosed as middle and late stage when they are already suffering abdominal distension,
abdominal pain, or pelvic tumor. This study aimed to comprehensively investigate the correlation of excision
repair cross complementary gene 1 (ERCC1) expression and the chemosensitivity of ovarian cancer.
Methods: A meta-analysis was conducted to comprehensively and quantitatively evaluate the relevant
research in this area. The literature published in PubMed, Web of Science, CQVIP, China National
Knowledge Infrastructure (CNKI), and Wanfang databases from establishment to June 2019 were searched.
The evaluation index of chemotherapy sensitivity was clinical effective rate (complete remission plus partial
remission). Two researchers independently screened the literature and extracted the data according to the
inclusion and exclusion criteria. Results: A total of 7 articles met the inclusion criteria, comprising 402 patients with ovarian cancer. The results showed that there was a significant difference in chemosensitivity between the low ERCC1 expression group and the high ERCC1 expression group ( $\mathrm{OR}=5.19$, 95\% CI: 3.15-8.54, $\mathrm{P}<0.01$ ), with the results of the ethnicity subgroup analysis being the same for the Asian and Caucasian populations.

Conclusions: The chemosensitivity of ovarian cancer patients with a low expression of ERCC1 is greater than that of patients with high expression.

Keywords: Excision repair cross complementary gene 1 (ERCC1); ovarian cancer; platinum-based chemotherapy; meta-analysis

Submitted Aug 05, 2020. Accepted for publication Sep 04, 2020.

doi: 10.21037/apm-20-1634

View this article at: http://dx.doi.org/10.21037/apm-20-1634

\section{Introduction}

Ovarian cancer is one of the most common malignant tumors in women, with the third highest incidence, second only to cervical cancer and endometrial cancer. Due to the lack of typical clinical symptoms and ineffective screening methods for early ovarian cancer, $60-70 \%$ of patients with ovarian cancer are diagnosed as middle and late stage when they are already suffering abdominal distension, abdominal pain, or pelvic tumor. At present, the main screening methods for ovarian cancer are color Doppler ultrasound, nuclear magnetic resonance imaging (NMR), computed tomography (CT), and tumor markers, but the specificity 
and sensitivity of these methods for ovarian cancer are low. Epithelial ovarian cancer (EOC) is one of the three most common malignant tumors in the female reproductive system. It has high mortality and poor prognosis, and seriously threatens the physical and mental health of women. The ovaries are located deep in the pelvis. Due to the lack of early diagnostic methods and inconspicuous clinical symptoms, most ovaries afflicted with cancer are only discovered in the late stage. According to some studies, about $70 \%$ of the patients are diagnosed in this stage (1). The 5 -year survival rate of patients with ovarian cancer has also hovered under $45 \%$ (2). The mortality of the cancer ranks first among gynecological tumors and poses a serious threat to the lives of women. The embryonic development, tissue anatomy, and endocrine function of the ovaries are complex, and the early symptoms are not typical. It is very difficult to differentiate between benign or malignant ovarian tumor tissue before operation (3). With the advance of medical science and technology in recent years, the progress of chemotherapy combined with paclitaxel and platinum has greatly improved the therapeutic effect and success rate of ovarian cancer treatments. Nevertheless, there has been no significant improvement to the 5 -year survival rate of ovarian cancer patients. A small portion of patients in the initial stage of treatment do not respond to platinum drugs, while the patients who are sensitive to the initial treatment experience recurrence. According to the literature, the 5-year survival rate of advanced ovarian cancer is $20-30 \%$, while that of early ovarian cancer is as high as $90 \%$ (4). Surgery combined with chemotherapy cannot cure advanced ovarian cancer and only offers temporary relief. Thus, $70-80 \%$ of patients with early ovarian cancer still experience recurrence (5). The resistance of tumor to platinum is the main obstacle. The development of tumor cell platinum resistance is caused by multiple factors, which may include the decrease of drug accumulation, the increase of glutathione level and metallothionein, and the improvement of DNA repair ability (6). Nucleotide excision repair cross complementary gene 1 (ERCC1) is a gene that can recognize DNA damage and cleave DNA chain. Its overexpression can remove platinum DNA compounds, and it has been demonstrated that damaged tumor DNA can be repaired and replicated again (7). There is a correlation between the high expression of ERCC1 and the chemosensitivity of NSCLC platinum drugs (8). The ERCC1 gene is thought to be able to repair platinumdamaged tumor cell and DNA compounds, which are critical to the ability of tumor tissue to restore replication and proliferation (9). Therefore, its expression may be used to predict the efficacy of platinum chemotherapy in patients with EOC, so as to guide the clinical use of drugs (10). In order to investigate the relationship between different gene expression levels of ERCC1 and platinum chemosensitivity in patients with ovarian cancer, we conducted a metaanalysis to comprehensively and quantitatively evaluate the relevant research in this area. We present the following article in accordance with the PRISMA reporting checklist (available at http://dx.doi.org/10.21037/apm-20-1634).

\section{Methods}

\section{Literature retrieval}

A literature search concerning the expression level of ERCC1 and the chemotherapy of ovarian cancer was conducted on the PubMed, Web of Science, CQVIP, China National Knowledge Infrastructure (CNKI), and Wanfang databases from the establishment of the databases to June 2019. The following keywords in Chinese and English language were used: "ovarian cancer", "ovarian carcinoma", "ERCC1", "excision repair cross-complementation group 1 ", "chemotherapy", and other variations.

\section{Literature screening and data extraction}

After two trained researchers independently read the titles and abstracts, excluding trials that obviously did not meet the inclusion and exclusion criteria, they further read the full text of the potentially eligible studies to further determine whether they met the requirements and crosschecked the reference lists. For trials where decisions on inclusion were difficult or where there were divergent opinions, inclusion was ultimately resolved through panel discussions. The two researchers independently extracted the information according to the predesigned data extraction table. For the repeatedly published articles, only the most recently published and the most complete articles were selected. For Chinese literature, research entered by the Peking University Library or the Key Magazine of China Technology was chosen. According to the Newcastle-Ottawa scale (NOS) (11), the quality of the literature was evaluated. Literature with fewer than six stars was considered low-quality while literature with six stars or more was considered high-quality. Only high-quality articles were included in this study. 


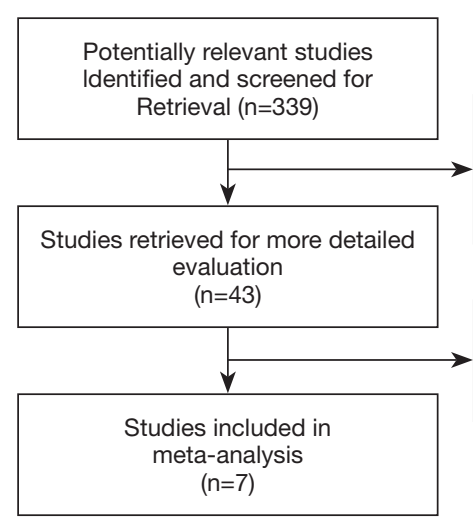

Figure 1 A flow diagram of the study selection process.

\section{Inclusion and exclusion criteria}

Literature included in the meta-analysis were required to have the following content: patients with ovarian cancer diagnosed by pathological or cytological examination; expression of ERCC1 in ovarian cancer tissues detected by immunohistochemistry (IHC); the same chemotherapeutic drugs used in patients regardless of positive or negative expression of ovarian cancer; detailed clinical data; original data on the number of positive cases or the positive rate. Repeated literature reports on the same study and animal studies were excluded.

\section{Statistical methods}

The meta-analysis was carried out by using stata 15.0 software. The odds ratios (ORs) and $95 \%$ confidence intervals (CIs), as the effect size, were calculated to represent the results. Q test was used to assess the heterogeneity of the results of each study. If $\mathrm{I}^{2} \geq 50 \%$, or $\mathrm{P} \leq 0.05$, the results were considered heterogenous, and the random effects model (REM) was used. If $\mathrm{I}^{2}<50 \%$, and $\mathrm{P}>0.05$, heterogeneity was considered absent, and so the FEM could be used for data consolidation. $Z$ test was used to assess the significance of the combined $O R$ value. This meta-analysis was included in the evaluation of publication bias, and the judgement standard was whether the funnel plot was symmetrical or not. The funnel plot used the standard error of each study $\log (\mathrm{OR})$ to map its OR value. If the funnel plot was asymmetrical, the presence of publication bias was considered possible, and Egger's Test was used to test the publication bias.
Exclude $(n=296)$ :

Title review not meet purpose $(n=247)$

Not relevant to chemothoerapy $(n=36)$

Meeting abstracts $(n=13)$

Exclude $(\mathrm{n}=35)$ :

Review article $(n=31)$

Overlapped data $(n=4)$

\section{Results}

\section{Literature retrieval results}

A comprehensive search of each database was completed, and the results from the literature were cross-checked. Each article was screened in strict accordance with the requirements of inclusion and exclusion criteria and quality control. The specific screening process is displayed in Figure 1, and the basic characteristics of the literature are summarized in Table 1. Ultimately, seven studies (12-18) were included in the analysis, which comprised a total of 402 patients positive for ovarian cancer, with a ERCC1 positive expression rate of $53.2 \%$.

\section{Relationship between ERCC1 expression and chemosensitivity of platinum drugs in ovarian cancer}

The meta-analysis showed that there was no statistical heterogeneity among the seven studies $\left(\mathrm{P}=0.935, \mathrm{I}^{2}: 0.0 \%\right)$. The FEM was used for analysis, and the forest plot was subsequently drawn (Figure 2). The complete remission plus partial remission rate of ovarian cancer patients reported in the seven studies (402 cases) indicated that the patients with a low expression of ERCC1 were more sensitive to chemotherapy than those with a high expression. The combined OR value was 5.19 (95\% CI: 1.18-9.11, $\mathrm{P}<0.01$ ). The results of ethnicity subgroup analysis showed that the low expression of ERCC1 was associated with $\mathrm{OR}=5.31$ (95\% CI: $1.18 \pm 9.11, \mathrm{P}<0.01)$, and that the Caucasian population was associated with $\mathrm{OR}=4.46$ (95\% CI: $1.18 \pm 16.91, \mathrm{P}<0.028)$. The differences were statistically significant (Table 2). The results showed that there was 
Table 1 Characteristics of included studies

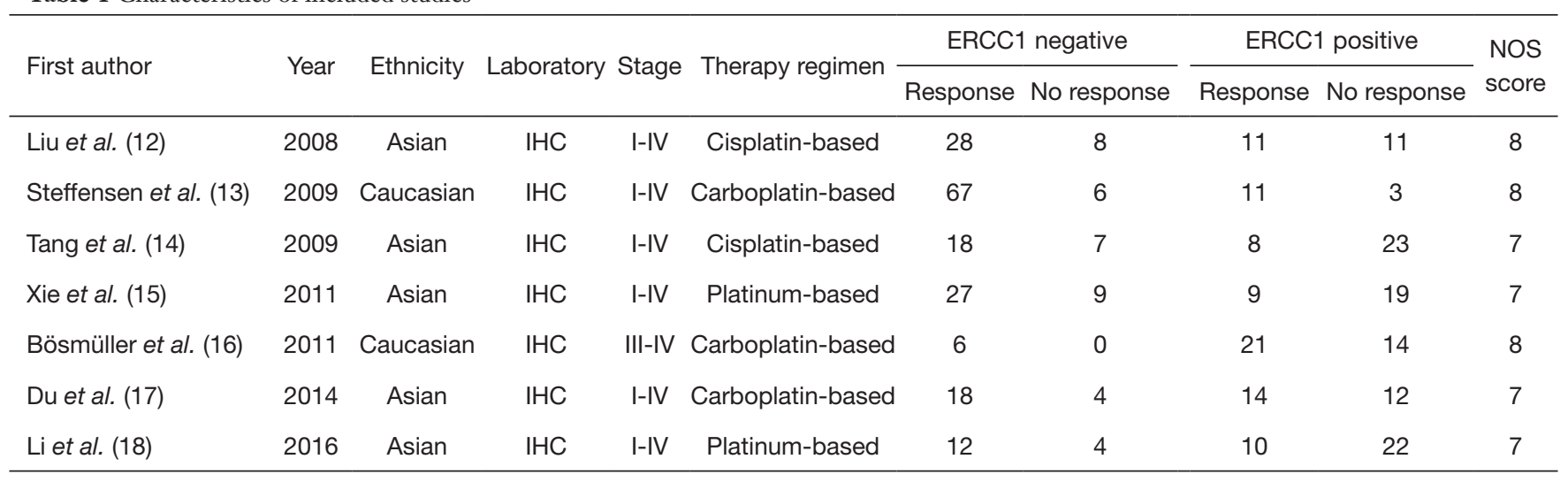

ERCC1, excision repair cross complementary gene 1; IHC, immunohistochemistry.

\begin{tabular}{|c|c|c|c|c|}
\hline \multirow{2}{*}{$\begin{array}{l}\text { Study } \\
\text { Asian }\end{array}$} & \multicolumn{2}{|l|}{ Year } & \multirow[t]{2}{*}{ OR $(95 \% \mathrm{Cl})$} & $\begin{array}{l}\% \\
\text { Weight }\end{array}$ \\
\hline & & & & \\
\hline GYLiu & 2008 & $\rightarrow+1$ & $3.50(1.11,11.02)$ & 18.82 \\
\hline Lin Tang & 2009 & $\longrightarrow$ & $7.39(2.26,24.23)$ & 12.41 \\
\hline Cong Xie & 2011 & 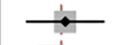 & $6.33(2.12,18.92)$ & 15.70 \\
\hline Pei Du & 2014 & $\because$ & $3.86(1.02,14.58)$ & 14.47 \\
\hline $\mathrm{Li} \mathrm{Li}$ & 2016 & $\longrightarrow$ & $6.60(1.70,25.62)$ & 10.34 \\
\hline Xing-Lin Wang & 2017 & + & $8.33(3.00,23.17)$ & 15.75 \\
\hline \multicolumn{2}{|c|}{ Subtotal $(\mathrm{l}$-squared $=0.0 \%, P=0.875)$} & & $5.86(3.64,9.43)$ & 87.49 \\
\hline Caucasian & 2009 & & $3.05(0.66,14.00)$ & 9.41 \\
\hline Bosmuller & 2011 & & $8.77(0.46,167.94)$ & 3.10 \\
\hline \multicolumn{2}{|c|}{ Subtotal $(\mathrm{I}$-squared $=0.0 \%, P=0.506)$} & & $4.46(1.18,16.91)$ & 12.51 \\
\hline \multicolumn{2}{|c|}{ Overall (I-squared $=0.0 \%, P=0.923$ ) } & \rangle & $5.68(3.63,8.89)$ & 100.00 \\
\hline & & & & \\
\hline
\end{tabular}

Figure 2 Forest plot for the association between ERCC1 expression and platinum chemosensitivity of ovarian cancer. ERCC1, excision repair cross complementary gene 1 .

Table 2 Meta-analysis between ERCC1 expression and platinum chemosensitivity of ovarian cancer

\begin{tabular}{llcccccc}
\hline Items & $\mathrm{n}$ & $\mathrm{OR}$ & $95 \% \mathrm{Cl}$ & $\mathrm{P}$ & $\mathrm{I}^{2}$ & $\mathrm{P}$ for heterogeneity & Publication bias \\
\hline Overall & 8 & 5.68 & $3.63-8.89$ & 0.000 & 0.00 & 0.923 & 0.815 \\
Caucasian & 2 & 4.46 & $1.18-16.91$ & 0.028 & 0.00 & 0.506 & $\mathrm{NA}$ \\
Asian & 6 & 5.86 & $3.64-9.43$ & 0.000 & 0.00 & 0.875 & 0.361 \\
\hline
\end{tabular}

ERCC1, excision repair cross complementary gene 1; OR, odds ratio; $\mathrm{Cl}$, confidence intervals. 


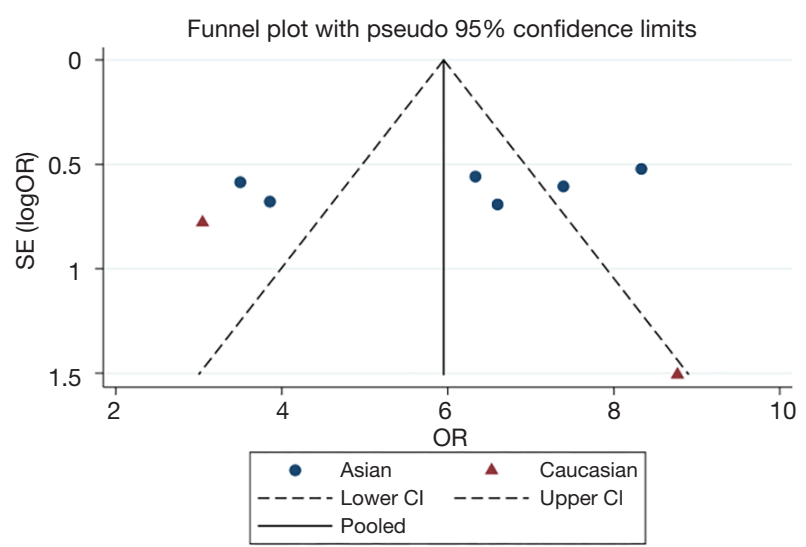

Figure 3 Funnel plot for the assessment of publication bias.

a correlation between the expression of ERCC1 and platinum sensitivity in patients with ovarian cancer. The chemotherapy sensitivity of patients with a low expression of ERCC1 was higher than that of patients with a high expression of platinum.

\section{Publication bias analysis}

A funnel plot was used to analyze the selected literature, and its symmetry was analyzed by Egger's $Z$ test. The specific results are shown in Table 2 and Figure 3. The results demonstrated that the funnel plot was basically symmetrical, and according to Egger's Test, the P value was more than 0.05 , indicating no publication bias.

\section{Sensitivity analysis}

The results of sensitivity analysis are summarized in Figure 4. After excluding each study one by one and conducting the meta-analysis, the results suggested there to be no significant change in the combined effect, indicating that the seven articles included were reliable.

\section{Discussion}

The incidence of ovarian malignant tumors is second only to cervical cancer and endometrial cancer among gynecological malignant tumors, and the 5-year survival rate is only $20 \%$ to $30 \%$ (19). The mortality rate of this disease is so high in part because of the lack of simple and specific early screening criteria and the resistance of tumors to chemotherapeutic drugs. Platinum preparation is the first-line chemotherapy drug for the treatment of ovarian cancer. It inhibits tumor cell division by inhibiting DNA replication (20). The ERCC1 gene is a DNA damage repair gene. Its function involves repairing the genome dysfunction caused by carcinogenic factors which has the effect of inhibiting tumor growth. However, it has been reported that the ERCC1 gene is involved in the repair of DNA damage caused by platinum drugs which thus reduces the efficacy of chemotherapy. The expression level of the ERCC1 gene in tumor is closely related to the chemotherapeutic effect platinum drugs have on patients (21). ERCC1 has endonuclease activity, which is the core factor of nucleotide excision repair (NER) pathway and which plays an important role in the repair process. It is essential for the in vivo repair process, as it maintain two functions, excision of 5 ' end and damage identification. On the one hand, ERCC1, as an endonuclease at the protein level, can repair the DNA damage caused by platinum drugs. On the other hand, the mechanism of drug resistance caused by the high expression of ERCC1 in tumor cells leads to the damage of DNA. These countervailing phenomena can cause platinum resistance. Platinum enters tumor cells and hydrolyzes into dichlorodiaminoplatinum, which binds to intracellular nucleophilic DNA to form platinum-DNA adducts, resulting in interchain or intrachain crosslinking of DNA, affecting the replication and transcription of DNA. It has the ultimate effect of damaging DNA and precipitating the apoptosis of tumor cells.

ERCC1 can recognize and repair the injury caused by platinum. It can effectively remove the damaged nucleotides in time, so that the tumor cells themselves can avoid the effect of chemotherapeutic drugs and produce drug resistance, thus affecting the chemosensitivity of platinum drugs. The expression of ERCC1 is also related to the occurrence and prognosis of ovarian cancer. The expression of ERCC1 in 64 cases of epithelial ovarian carcinoma was studied (22). It was found that the positive expression rate of ERCC1 in the chemotherapy-resistant group was significantly higher than that in the chemotherapy-sensitive group. Furthermore, the expression of ERCC1 was found to not be related to clinical characteristics. In a study of 60 cases of ovarian tumors (23), it was observed that the positive expression of ERCC1 in borderline ovarian tumors was significantly higher than that in benign ovarian tumors and ovarian cancer. The expression of ERCC1 was not correlated with age, pathological type, tumor differentiation, or International Federation of Gynecology and Obstetrics (FIGO) stage. It has been reported that 


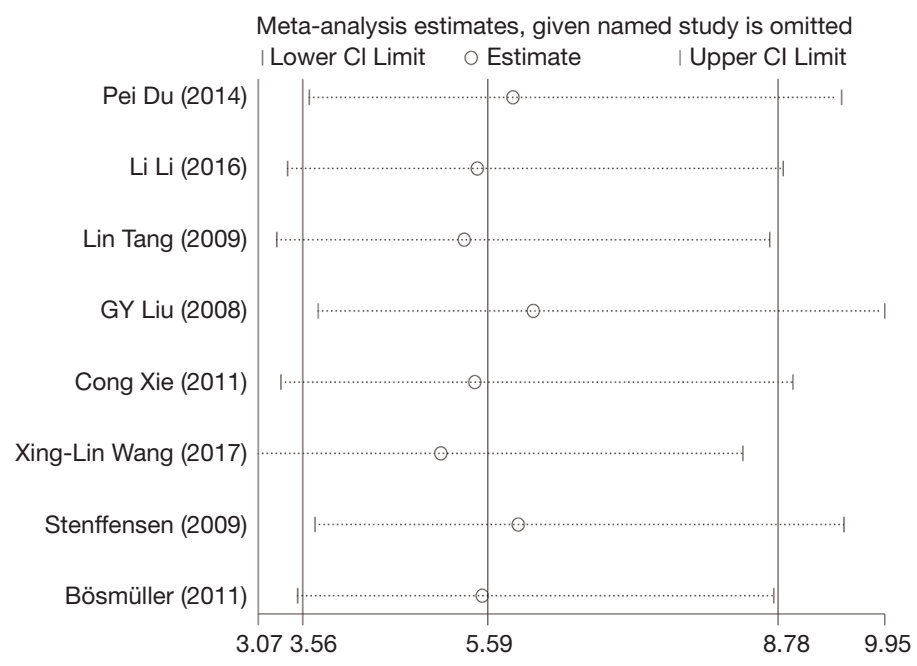

Figure 4 Sensitivity analysis results.

there is a correlation between high expression of ERCC1 and cisplatin resistance, which also affects the survival rate of patients with ovarian cancer $(13,24)$. Current studies tend to suggest that people with a low expression of ERCC1 have a better response to cisplatin chemotherapy of ovarian cancer than those with high expression (25). However, the conclusion is also controversial. Bösmüller (16) and others have found that the expression of ERCC1 is not related to platinum chemosensitivity in patients with ovarian cancer. However, it is important to note that research into the expression of the ERCC1 gene protein is limited to a small number clinical studies with small sample sizes, with only a few having large samples. Therefore, we conducted a meta-analysis of the research that examined the relationship between the expression of ERCC1 gene protein and platinum chemosensitivity in patients with ovarian cancer, and comprehensively evaluated the effect of ERCC1 expression on platinum chemosensitivity in patients with ovarian cancer.

Another purpose of the study is to provide evidencebased theory for clinical treatment. There have been many related studies on the relationship between ERCC1 gene expression and the sensitivity of platinum-based chemotherapy in patients with ovarian cancer, but the results of these studies are not uniform. Studies have shown that ovarian cancer patients with positive ERCC1 protein are prone to platinum drug resistance $(26,27)$. However, some studies have shown that ERCC1 protein expression has no significant correlation with the prognosis of ovarian patients with platinum chemotherapy (22).
The results of this study showed that the overexpression of ERCC1 was correlated with the chemosensitivity of platinum drugs in ovarian cancer. Compared with the high expression of ERCC1, low expression was more sensitive to platinum chemotherapy for ovarian cancer. In the subgroup analysis, the Asian and Caucasian populations yielded the same results. From the point of view of statistical heterogeneity, there was no statistically significant difference, indicating that the homogeneity of samples in the study was good. From the perspective of publication bias, the funnel plot was basically symmetrical. Egger's Test yielded a $\mathrm{p}$ value greater than 0.05 , indicating no publication bias was present. The results of sensitivity analysis, which was performed by excluding each study one by one and using meta-analysis, did not show a significant change in the combined effect, indicating that the stability of the included studies was acceptable. The conclusion of the present metaanalysis was therefore considered reliable. Our results were consistent with those of Liu et al. (25) who demonstrated that those ovarian cancer patients with a low expression of ERCC1 were more sensitive to platinum chemotherapy.

Naturally, our study has some limitations. First, the methods used to detect the expression of ERCC1 in the included studies were all basically immunohistochemical in nature, and thus there might have been some subjectivity and inconsistency in judgment across the different researchers. Furthermore, the number of included studies was relatively small; most were from Asian countries, only two were from Europe and North America, and none were from African or South American countries. 


\section{Conclusions}

The meta-analysis revealed a correlation between ERCC1 overexpression and platinum chemosensitivity of ovarian cancer. Low expression of ERCC1 is more sensitive to platinum chemotherapy of ovarian cancer than high expression, and this is true for both Asian and Caucasian populations. ERCC1 status may thus be a potential biomarker for predicting the efficacy of platinum chemotherapy in ovarian cancer. However, in view of the limitations of this study, large-scale and well-designed studies are still needed to investigate the factors that may affect the response to platinum chemotherapy.

\section{Acknowledgments}

Funding: None.

\section{Footnote}

Reporting Checklist: The authors have completed the PRISMA reporting checklist. Available at http://dx.doi. org/10.21037/apm-20-1634

Conflicts of Interest: All authors have completed the ICMJE uniform disclosure form (available at http://dx.doi. org/10.21037/apm-20-1634). The authors have no conflicts of interest to declare.

Etbical Statement: The authors are accountable for all aspects of the work in ensuring that questions related to the accuracy or integrity of any part of the work are appropriately investigated and resolved.

Open Access Statement: This is an Open Access article distributed in accordance with the Creative Commons Attribution-NonCommercial-NoDerivs 4.0 International License (CC BY-NC-ND 4.0), which permits the noncommercial replication and distribution of the article with the strict proviso that no changes or edits are made and the original work is properly cited (including links to both the formal publication through the relevant DOI and the license). See: https://creativecommons.org/licenses/by-nc-nd/4.0/.

\section{References}

1. Cao Y, Ni X, Wang Y, et al. Clinical and prognostic significance of combined plasma fibrinogen concentrations and the monocyte-to-lymphocyte ratio in patients with ovarian cancer. Ann Transl Med 2019;7:242.

2. Webb PM, Jordan SJ. Epidemiology of epithelial ovarian cancer. Best Pract Res Clin Obstet Gynaecol 2017;41:3-14.

3. Yoshida A, Derchain SF, Pitta DR, et al. Comparing the Copenhagen Index (CPH-I) and Risk of Ovarian Malignancy Algorithm (ROMA): Two equivalent ways to differentiate malignant from benign ovarian tumors before surgery? Gynecol Oncol 2016;140:481-5.

4. Xue C, Zhu D, Chen L, et al. Expression and prognostic value of PD-L1 and PD-L2 in ovarian cancer. Transl Cancer Res 2019;8:111-9.

5. Miller KR, Patel JN, Zhang Q, et al. HOXA4/HOXB3 gene expression signature as a biomarker of recurrence in patients with high-grade serous ovarian cancer following primary cytoreductive surgery and first-line adjuvant chemotherapy. Gynecol Oncol 2018;149:155-62.

6. Reed E. Platinum-DNA adduct, nucleotide excision repair and platinum based anti-cancer chemotherapy. Cancer Treat Rev 1998;24:331-44.

7. Friboulet L, Olaussen KA, Pignon JP, et al. ERCC1 isoform expression and DNA repair in non-small-cell lung cancer. N Engl J Med 2013;368:1101-10.

8. Li G, Cheng D. Meta-Analysis of ERCC1 Protein Expression and Platinum Chemosensitivity in Non-SmallCell Lung Cancer. Evid Based Complement Alternat Med 2020;2020:7376568.

9. Simon GR, Sharma S, Cantor A, et al. ERCC1 expression is a predictor of survival in resected patients with nonsmall cell lung cancer. Chest 2005;127:978-83.

10. Deng Q, Yang H, Lin Y, et al. Prognostic value of ERCC1 mRNA expression in non-small cell lung cancer, breast cancer, and gastric cancer in patients from Southern China. Int J Clin Exp Pathol 2014;7:8312-21.

11. Stang A. Critical evaluation of the Newcastle-Ottawa scale for the assessment of the quality of nonrandomized studies in meta-analyses. Eur J Epidemiol 2010;25:603-5.

12. Liu GY, Qu QX, Mi RR, et al. [Relationship between nucleotide excision repair gene ERCC1 and resistance to cisplatin in ovarian cancer]. Zhonghua Zhong Liu Za Zhi 2008;30:184-7.

13. Steffensen KD, Waldstrøm M, Jakobsen A. The relationship of platinum resistance and ERCC1 protein expression in epithelial ovarian cancer. Int J Gynecol Cancer 2009;19:820-5.

14. Tang L, Liang J, Li YJ. Relationship between ERCC1 expression and cisplatin resistance in epithelial ovarian cancer. QILU YIXUE ZAZHI 2009:388-90. 
15. Xie C, Yin RT, Li YL, et al. [The protein expression of ERCC1 and survivin in epithelial ovarian carcinoma and their clinical significance]. Sichuan Da Xue Xue Bao Yi Xue Ban 2011;42:86-9.

16. Bösmüller H, Haitchi-Petnehazy S, Webersinke G, et al. Intratumoral lymphocyte density in serous ovarian carcinoma is superior to ERCC1 expression for predicting response to platinum-based therapy. Virchows Arch 2011;459:183-91.

17. Du P, Wang YF, Zhang XW, et al. Expression of ERCC1 and BRCA1 in patients with primary epithelial ovarian cancer. HERALD OF MEDICINE 2014;000:747-51.

18. Li L, Liu SN, Zheng WQ, et al. Expression and clinical significance of ERCC1 and tubb3 in ovarian cancer. Chinese Journal Of Clinical Medicine 2016;23:576-80.

19. Coleman RL, Monk BJ, Sood AK, et al. Latest research and treatment of advanced-stage epithelial ovarian cancer. Nat Rev Clin Oncol 2013;10:211-24.

20. Hildebrandt MA, Gu J, Wu X. Pharmacogenomics of platinum-based chemotherapy in NSCLC. Expert Opin Drug Metab Toxicol 2009;5:745-55.

21. Reed E. ERCC1 and clinical resistance to platinum-based therapy. Clin Cancer Res 2005;11:6100-2.

22. Rubatt JM, Darcy KM, Tian C, et al. Pre-treatment tumor expression of ERCC1 in women with advanced stage epithelial ovarian cancer is not predictive of clinical

Cite this article as: $\mathrm{Wu} \mathrm{J}, \mathrm{Li}$ R, Chen X, Chen C, Chen H, Ma B. Meta-analysis of excision repair cross complementary gene 1 (ERCC1) expression and platinum chemosensitivity in patients with ovarian cancer. Ann Palliat Med 2020;9(5):3428-3435. doi: 10.21037/apm-20-1634 outcomes: a Gynecologic Oncology Group study. Gynecol Oncol 2012;125:421-6.

23. Ju LL, Zhao CY, Ye KF, et al. Expression and clinical implication of Beclin1, HMGB1, p62, survivin, BRCA1 and ERCC1 in epithelial ovarian tumor tissues. Eur Rev Med Pharmacol Sci 2016;20:1993-2003.

24. Milovic-Kovacevic M, Srdic-Rajic T, Radulovic S, et al. Expression of ERCC1 protein in biopsy specimen predicts survival in advanced ovarian cancer patients treated with platinum-based chemotherapy. J buon 2011;16:708-14.

25. Liu SC, Lin H, Huang CC, et al. Prognostic role of excision repair cross complementing- 1 and topoisomerase-1 expression in epithelial ovarian cancer. Taiwan J Obstet Gynecol 2016;55:213-9.

26. Lin K, Ye D, Xie X. Protein expression levels of excision repair cross-complementation group 1 and xeroderma pigmentosum D correlate with response to platinum-based chemotherapy in the patients with advanced epithelial ovarian cancer. Int J Gynecol Cancer 2008;18:1007-12 .

27. Steffensen KD, Waldstrøm M, Jeppesen U, et al. Prediction of response to chemotherapy by ERCC1 immunohistochemistry and ERCC1 polymorphism in ovarian cancer. Int J Gynecol Cancer 2008;18:702-10.

(English Language Editor: J. Gray) 